

\title{
OBC-WOA: Opposition-Based Chaotic Whale Optimization Algorithm for Energy Efficient Clustering in Wireless Sensor Network
}

\author{
Maddali M.V.M. Kumar ${ }^{1 *}$ \\ Aparna Chaparala ${ }^{2}$ \\ ${ }^{I}$ Department of Computer Science and Engineering, \\ Acharya Nagarjuna University, Guntur, AndraPradesh, India \\ ${ }^{2}$ Department of Computer Science and Engineering, \\ $R V R$ \& JC College of Engineering, Guntur, Andra Pradesh, India \\ * Corresponding author's Email: maddalikumar0710@ gmail.com
}

\begin{abstract}
A large number of small sensors in the Wireless Sensor Network (WSN) can be an efficient tool for data collection in a range of environments. Each sensor transfers the information to the basic unit that transmits the information to the end user. The objective of clustering is to separate the network into sectors with a cluster head $(\mathrm{CH})$. The job is to collect, aggregate and transmit cluster heads to the base station. Energy Efficient Clustering has been used widely for energy conservation in wireless sensor networks (WSNs) and also preserves the restricted energy resources of their sensors. Cluster heads $(\mathrm{CHs})$ perform a major part and exhaust power faster than other member nodes in a distributed WSN. Opposition-based Chaotic Whales Optimization algorithm (OBC-WOA) is meta-heuristic optimization algorithm which has recently been proposed in opposition. It simulates humpback whales ' social behaviour. OBC-WOA produces randomly its population during exploration and exploitation stages, like other population-based systems, which can produce values far from the optimal alternative or block the development of local optima. The revised algorithm known as Opposition-based Chaotic Whale Optimization Algorithm (OBC-WOA) is designed to increase solution precision and reliability. The OBC-WOA uses a technique based on opposition to improve the efficiency of OBC-WOA. The OBC-WOA is screened with the initial WOA algorithm and other meta-heuristic techniques. The performance of the proposed approach is evaluated in terms of energy consumption, throughput, packet delivery ratio and network life time. When compared with the existing method the proposed method is $12 \%$ better than Whale Optimization algorithm (WOA), 25\% better than Gravitational Search Algorithm (GSA), 40\% better than Particle Swarm Optimisation (PSO) and 8\% better than Fuzzy K-Means and Centralized Mid-point Algorithm (FKM-CMA).
\end{abstract}

Keywords: Wireless sensor network (WSN), Cluster head $(\mathrm{CH})$, Opposition-based chaotic whale optimization (OBC-WOA), Energy efficient clustering, Exploration and exploitation.

\section{Introduction}

The evolving technology Wireless Sensor Networks (WSN) has seen an enduring surge in employs [1]. The Wireless Sensor Network (WSN) is an extremely distributive network that provides more features and is more self-organized [2].

Data aggregation is a necessary method for transferring the information to the base station because of the high correlations between the data obtained at various node levels [3]. A powerful technology for the energy conservation of sensor nodes is a clustering of sensor nodes [4]. In order to overcome network researchers ' deficiencies, unique nodes called' gateways' have a feature comparable to that of detectors. The cluster centres [5] have the function these gateways operate. Chaos was used in multiples meta-heurist algorithms to enhance efficiency, leading to a stronger velocity of convergence and prevention of local Optima Placing [6]. Efficient clustering in Wireless Sensor Networks (WSNs) [7] is a well-documented NDhard optimization problem. Various computational 
intelligence technologies have been implemented for energy-efficient WSN clusters [8].

In this study, we are proposing an opposition chaotic whale optimization (OBC-WOA) algorithm which enhances initial WOA efficiency through the introduction of opposition-based training to effectively approach the closeness of test candidates to global optimal local chaotic search for search areas. In addition, an energy-efficient OBC-WOA meta-heuristic clustering protocol is presented. The findings of simulations indicate that the clustering protocol is able to perform other well-known procedures as efficiency metrics based on packet distribution, throughput, power usage, network service life and latency.

The remaining article is structured as defies: Section 2 describes the field energy efficient clustering in WSN provides various works pertaining to the use of meta-heuristic algorithms. Section 3 describes the proposed methodology, illustrates the Network model and describes the Energy Efficient Clustering in Wireless Sensor Network. Section 4 illustrates the proposed Opposition-Based Chaotic Whale Optimization Algorithm (OBC-WOA). Section 5 presents the simulation results. Section 6 defines the conclusion and future scope.

\section{Literature survey}

There are several study projects in the literature, which use optimisation algorithms to determine the objectives for several tasks. A part of the work is examined in this section.

A number of clustering algorithms connected with the lately established WSN were presented. Zaatouri et.al [9] proposed two kinds of cluster algorithms such as homogeneous and heterogeneous. In these algorithms energy consumption constituted the major problem that caused too much trouble with the networks. The algorithm suggested by V. Saranya, et al., [10] was a stronger portable sinkbased WSN. The Energy Efficient Clustering Scheme (EECS) algorithm exceeded the durability and performance of the Manual-Gear (M-GEAR) and Modified-Leach (MOD-LEACH) Protocol for increasing the lifetime of the wireless sensor network. The plan also withstood the problem of the power gap and the issue of HOT SPOT. However some dissemination nodes were brought extra overhead in this technique.

Q. Wang et al., [11] they aimed to make the system efficient, so that the life of a wireless sensor network in the backdrop of big data could be extended in an effective manner. The drawback of the method was that, the network topology was required to keep unchanged over time, and sensor nodes were deployed uniformly. To reduce the energy consumption and to make the system efficient, an energy-efficient routing and clustering protocols centred on Particle Swarm Optimisation (PSO) have been provided in the Metal by $\mathrm{M}$. Azharuddin, and P. K. Jana [12]. The routing algorithm created a compromise between effectiveness and power equilibrium, whereas the clustering of the gates and detector nodes worries about energy faults. The drawback of the method was that, there exist a transient failure across the gateways and also the computation load was included on the base station (BS). So, the network was unable to self-configure and self-heal.

P. Lalwani, S. Das, H. Banka, and C. Kumar [13] Selection and routing in WSNs were computerpriced functions related to elevated network-level complexity. The demerit was that, the CRHS algorithm could not considered the fault-tolerance and delay for forwarding the data packets to the base station. To fix the issues indicated, the harmony search algorithm was used by O. M. D. Alia [14]. Results of the simulation showed that, during the simulations, an optimum number of clusters could be generated in each round. The drawback was that, the method affected the performance of clustering based WSN protocols.

Zahedi et al., [15] presented an innovative method for decreasing the amount of emails sent in vibrant systems and their effectiveness compared to LEACH algorithm were assessed. The reservation process initially improved the energy consumption. And, the overall energy was reduced because every node has data about its cluster head or non-cluster head-node. But the delay was increased in this method. S. Dutt et al., [16] has critically examined and created an altered protocol called Cluster-Head Restricted Energy Efficient Protocol (CREEP), to enhance the network life. But there was needed a trade-off between higher lifetime and throughput and also the system possessed the less energy efficiency.

N. Srikanth, and M.S.G. Prasad [17] proposed an Energy Efficient Multi-hop Routing Clustering Protocol (EEMRCP) based on Fuzzy K-Means and Centralized Mid-point Algorithm (FKM-CMA) which was used for network lifetime improvement. To improve K-means algorithm, a Midpoint method in initial centroid selection was used. However the optimization problem still existed in this technique. 


\subsection{Problem definition}

From the literature survey, we mainly focused on the network lifetime, energy consumption and the performances of the network. The main drawbacks of the existing methods such as high energy consumption, less network life time, large delay and less energy efficiencies are overcome by the proposed method by the use of a comprehensive energy-efficient chaotic whale optimization routing algorithm and thus the OBC-WOA's shows superior output because of the chaotic maps in the search space.

\section{Proposed methodology}

\subsection{Network model}

The model for the network is regarded a model of free space. It is equipped with a transmitter and a receiver. The following characteristics are assumed for WSN as:

- All sensor nodes are randomly installed and stationary.

- All nodes are uniform and energy restricted.

- The BS can be placed in or outside the screening region and is stationary.

- Each server periodically collects the information and always has certain information to forward.

- Nodes understand neither their precise roles nor other nodes ' location.

- The nodes are self-organized and do not have to be controlled after use.

- The fusion of information is used to minimize the complete volume of transmitted data.

- Each node can function as the top of a cluster. All the above properties and limitations of the WSN scenario for simulation are considered. By comparing the signal power obtained, the nodes can calculate the distance between the BS and other nodes.

\subsection{Energy efficient clustering in wireless sensor network}

The primary objective of hierarchical routing or cluster oriented routing is to keep the energy effectively during multi-hop communication. The procedure enables the head of a cluster to contact the base station directly. The nodes in the cluster presents the base station and separate it from it, recognizing the signal transmitted during the network use phase from the base station at a certain

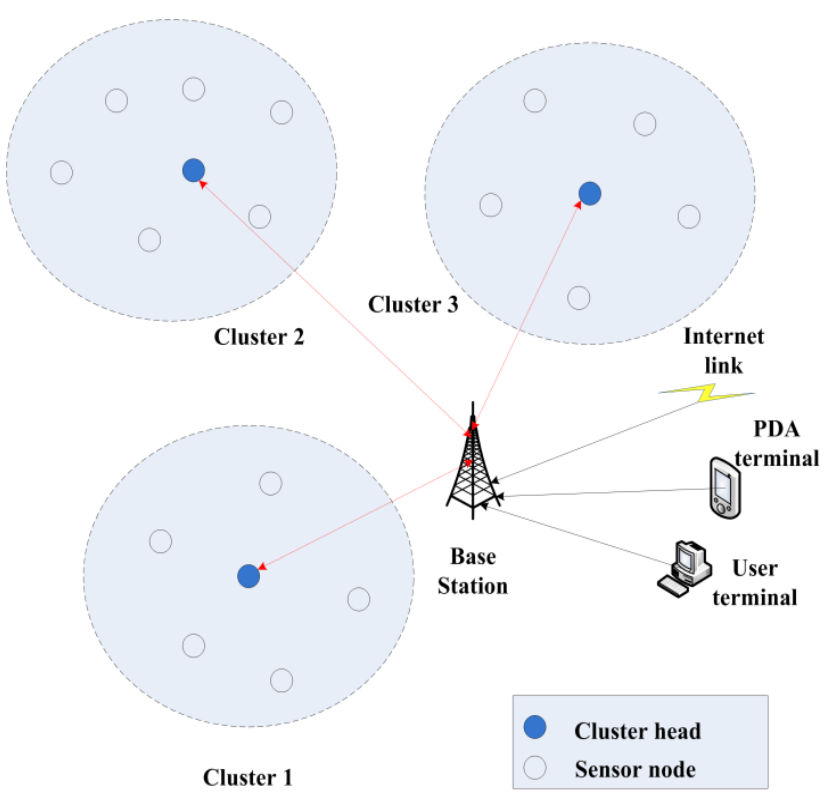

Figure. 1 Cluster of WSN

power level. The suggested approach consists of three measures: 1) creation of clusters 2) choice of cluster heads and 3) transmitting data. Clusters formation is usually based on the energy reserve near the cluster head of sensors. For WSN energy conservation, clustering functions a significant part. Energy usage, network survival and interoperability can be enhanced with clustering in WSNs. Since the routing task is only mandated for cluster heads per cluster, while the other sensor nodes only pass data on to cluster heads. In high-density sensor networks, clustering provides major applications because the management of a number of cluster members (cluster heads) in each cluster is easier than the administration of whole sensor nodes. In WSNs, the sensor nodes are restricted in terms of a resource, which implies that they have reduced energy, memory, storage and computer capacities. The energy produced by the sensor nodes is the key source of energy depletion in sensor nodes to pass information from the sensor nodes to the base station. The cluster of WSN is shown in Fig. 1.

\subsubsection{Comparison of the proposed methodology with the existing energy efficient clustering techniques}

The Performance estimation of the proposed approach attains higher throughput, network lifetime and packet delivery ratio as well as reduced energy consumption and delay as compared to the existing methods such as Energy Efficient Magnetic resonance cholangio pancreatography( EEMRCP) using Fuzzy $\mathrm{K}$ means and centralized mid-point algorithm for network lifetime improvement in WSNs, Energy Efficient Clustering Scheme (EECS) 
method for WSN to improve the durability and performance, and Spectral partitioning and fuzzy cmeans based clustering to improve the network lifetime etc.

\subsubsection{Creation of clusters}

The clustering process establishes the group of nodes below the head of the cluster. In the clustering protocols, the method of cluster formation and the number of nodes is very critical. Clustering demonstrates to be an efficient system for the leadership of the sensor clusters by increasing the lifetime of their clusters. The originator will be indicated at the beginning of each process and the messages will be transmitted using different cluster organisation techniques.

The anticipated network lifespan can be assessed using the complete network energy accessible divided by the complete energy used in each round. It therefore means, all network nodes will have the same lifespan and the stable operating duration and will be expanded if all clusters have such anticipated life-time for the network. We suppose that an $\mathrm{N}$ node WSN is available. In the case of node $k$ it offers energy for $E N_{0}\left(1+x_{k}\right)$ with a traffic load for $T R L_{0}\left(1+y_{k}\right)$. The network is divided into $p$ clusters with $p k$ member nodes $p$ in ' $k$ ' cluster. In addition, we denote the range from the nodes to the $\mathrm{CH}$ as $\operatorname{dist}\left(n d_{k}, \mathrm{CH}_{K}\right)$ and the range from $\mathrm{CH}$ to the base as $\operatorname{dist}\left(\mathrm{CH}_{K}, \mathrm{BS}\right)$. The total energy of a residual cluster $E N\left(C_{k}\right)$, can be used for complete consumption of energy by Eq. (1).

$$
\begin{aligned}
& E N\left(C_{k}\right)=\sum_{p_{k}}\left(T R L _ { 0 } ( 1 + y _ { k } ) \left(e n_{t}+\right.\right. \\
& \left.\left.\operatorname{cdist}^{n}\left(\operatorname{lnd}_{k}, C H_{k}\right)\right)\right)+\left(e n_{r} \sum_{p_{k}}\left(T R L_{0}\left(1+y_{k}\right)\right)+\right. \\
& \operatorname{EN}_{D A} \sum_{p_{k+1}}\left(T R L_{0}\left(1+y_{k}\right)\right)+T R L_{0}\left(e n_{t}+\right. \\
& \left.\operatorname{Edist}^{n}\left(C_{k}, B S\right)\right)
\end{aligned}
$$

$E N\left(C K_{k}\right)$, Energy usage is comprised of four components, as provided in Eq. (1): (i) node information sent to $\mathrm{CH}$, (ii) $\mathrm{CH}$ information received from member nodes, (iii) $\mathrm{CH}$ information aggregate obtained, and (iv) $\mathrm{CH}$ information transfers aggregate to a source. The load of traffic has a significant function to perform in Eq. (1)

For Cluster $k$, the ratio of the total cluster residual energy, $E N_{c}(k)$ and total cluster traffic load $T R L_{c}(k)$ is defined by a new parameter called Energy Consumed by the Transceiver (ETR) and is given as follows:

$$
E T R_{k}=E N_{c}(k) / T R L_{c}(k)
$$

Each $\mathrm{CH}$ monitors, when chosen for $\mathrm{CH}$ _MSG, the function of the CH_MSG and allows the membership of nodes other than the CH_MSG. This CH_MSG message contains data from the CH ETR, which makes it important to identify which $\mathrm{CH}$ should be included because a non- $\mathrm{CH}$ node can obtain more than one CH_MSG. The proximity metric between a member node and its corresponding $\mathrm{CH}$ is probably essential for energy conservation reasons and should consequently be taken into account. In addition to energy saving, the ETR equilibrium also requires to be taken into account for the permanent expansion. During clustering, control messages are used. Further messages of control require greater energy consumption. Iteration would therefore be prevented and more energy can be preserved if the nodes can be grouped without iteration. If a node discovers that its own ETR is lesser than any CHs in energyefficient clustering (EEC), it is expected to assist to balance ETR. For the purpose specified in (3), threshold permeability $(\mu)$ is defined. The node takes into account only the CHs who's ETR fulfils the condition of (3). It adds at least the permeability factor, $\mu$ to the ETR equilibrium and then connects one with minimal range. If the node found that not all $\mathrm{CHs}$ were fulfilled in (3), then the $\mathrm{CH}$ would be connected with the closest range. The Energy Consumed by the Transceiver (ETR) of a non-CH node may be greater than or closer to the ETR with the $\mathrm{CH}$ node. This is shown in the below equation,

$\frac{E N_{C H}+E N_{n d(k)}}{T R L_{C H}+T R L_{n d(k)}}-\frac{E N_{C H}}{T R L_{C H}}>\frac{E N_{C H}}{T R L_{C H}} \mu$

Fewer $\mathrm{CHs}$ will be chosen after some nodes have died. The condition of (3) is met by a non- $\mathrm{CH}$ that receives less $\mathrm{CH} \_$MSGs and less $\mathrm{CH}$. The non$\mathrm{CH}$ enters the $\mathrm{CH}$ with minimum range when a node falls, for energy saving account. A node sends a JOIN _CLUSTER MSG to a $\mathrm{CH}$ and becomes a partner node of this node after determining which $\mathrm{CH}$ is to enter. If all nodes are grouped, the $\mathrm{CHs}$ create a timetable and deliver this signal to their partner nodes so that they are able to submit information in their corresponding slot.

\subsubsection{Choice of cluster heads}

The next stage is to pick leaders who will lead in each cluster. The next phase is cluster formation. Cluster heads are burdened by aggregating information and transmitting information from their cluster members to their base station. The cluster head's load rate is commensurate with the cluster 
size. The top of the cluster is randomly chosen or allocated by the network developer. The cluster top is chosen by random selection method, depending on the probability that the node was never chosen as the cluster head in the entire network life. The cost of working as cluster head over a single node is thus decreased by turning the accountability between all nodes in the cluster. The choice of the cluster head is rotating and takes place during a certain time span. During communication, the technique seeks to decrease energy use, since it attempts to position the cluster top in the cluster almost at other nodes.

We suppose a heterogeneous N-node multi-level WSN. The nodes have distinct primary energies and distinct loads of traffic. The first energy and traffic loads of sensors are allocated randomly over the closed set $\left[E N_{0}, E N_{0}\left(1+x_{\max }\right)\right]$ and $\left[T R L_{0}\right.$, $T R L_{0}\left(1+y_{\max }\right)$ ], where the basic energy node is $E N_{o}$ and also the lower bound of the initial energy and the value of the maximally initial energy are calculated by $y_{\max }$. Initially, the node $R_{k}$ has an original $E N_{o}\left(1+x_{k}\right)$ energy that is $x_{k}$ time as much energy as the $E N_{o}$ basic node energy and the $T L_{0}\left(1+y_{k}\right)$ traffic charge is $y_{k}$ times as large as the $T L_{0}$ basic traffic load. The WSN can be charged with equ (4) and (5) the complete original energy and the load of traffic:

$$
\begin{aligned}
& E N_{\text {tot }}=\sum_{k=1}^{N} E N_{0}\left(1+x_{k}\right) \\
& \quad=E N_{0}\left(N+\sum_{k=1}^{N} x_{k}\right. \\
& \quad T R L_{t o t}=\sum_{k=1}^{N} T R L_{0}\left(1+y_{k}\right)=T R L_{0}(N+ \\
& \left.\sum_{k=1}^{N} y_{k}\right) \\
& \overline{E N(q)}=E N_{\text {tot }}(q) / N \\
& \overline{T R L}=T R L_{\text {tot }} / N
\end{aligned}
$$

Then Eqs. (6) \& (7) are the median node energy for $q^{\text {th }}$ round and traffic charge during the stable era. The amount of $\mathrm{CHs}$ or cluster anticipated should be $N b_{\text {opt }}$ for a network with $\mathrm{N}$-nodes and with an optimized probability of $b_{o p t}$ for every node. In order to obtain greater energy efficiency in the network, this value must also be ensured in Energy Efficient Clustering (EEC). With regard to the remaining energy and traffic loads of the nodes shown in Eq. (8), the EEC sees the selection of CHs.

$$
b_{k}=x \times b_{o p t} \frac{E N_{q}(k)}{E N_{q}}-y \times b_{o p t} \frac{T R L_{q}(k)}{T R L_{q}}
$$

Where $x-y=1$ and this system ensures that the expected amount of $\mathrm{CHs}$ or clusters is guaranteed as demonstrated in Eq. (9).

$$
\begin{aligned}
& N \sum_{1<K<N} b_{k}=N \sum_{1<K<N}\left(x \times b_{o p t} \frac{E N_{q}(k)}{E N_{q}}-\right. \\
& \left.y \times b_{o p t} \frac{T R L_{q}(k)}{T R L_{q}}\right)=N b_{o p t}(x-y)=N b_{o p t}
\end{aligned}
$$

We thus obtain the probability limit, $Q\left(R_{k}\right)$, in order to determine if a node itself becomes $\mathrm{CH}$, as shown in Eq. (10), where $H$ is the number of nodes which can be $\mathrm{CHs}$ around $t$. If $R_{k}$ node throughout latest $1 / b_{k}$ rounds was not a $\mathrm{CH}$, node $R_{k}$ is a $H$. When node $\mathrm{Si}$ is found to be a $\mathrm{CH}$ at any round $t$, it selects a random number ranging from 0 to 1 . In each round $t$ is the node $R_{k}$ if it is eligible for a $\mathrm{CH}$. The $R_{k}$ node becomes a $\mathrm{CH}$ if the amount is lower than the limit.

$$
\begin{aligned}
& Q\left(R_{k}\right)= \\
& \left\{\begin{array}{cc}
\frac{b_{k}}{1-b_{k}\left(\bmod \left(t, \operatorname{round}\left(\frac{1}{b_{k}}\right)\right)\right)} & \text { if } R_{k} \in H \\
0 & \text { otherwise }
\end{array}\right.
\end{aligned}
$$

Each node gives a tiny packet of energy and traffic to the repository when the network begins. As the load of a node throughout the operating period is presumed to be constant, only the median energy needs to be improved at any time. The node supplies its remaining energy in the packet it gives to the $\mathrm{CH}$ during each round. Once all data are collected, $\mathrm{CH}$ sends the aggregate packet to the repository with the residual energy data from its nodes. Then the server quantifies the mean node energy throughout the network and transmits it to the nodes. For $\mathrm{CH}$ selection for the next round, the mean residual node value is used.

\subsubsection{Transmitting data}

The data can be transferred by the ordinary nodes to the respective cluster heads following the cluster formation and cluster heads selection. In this stage, the closest cluster head is attached to each ordinary node. Cluster heads shall be allocated to each cluster member to implement the TDMA criterion. In the assigned interval, each node sends its information in the shape of an information signal to cluster heads. After all emails from the nodes of the grid, the cluster members collect and convey information to the base station. Then all nodes will be calculated to consume energy. Distance of 
diffusion (dist) is contrasted with range limit (disto) and where range of transmission (dist) is less than dist $_{0}$ node energy usage is equal to dist $^{2}$ otherwise equivalent to $d i s t^{4}$. The complete energy required by an 1-bit data packet transmission device is applied by Eq. (11) and is shown as follows:

$$
\begin{aligned}
& E N_{\text {Trans }}(l, \text { dist })= \\
& \left\{\begin{array}{c}
e n_{r} \times l+\left(e n_{t}+\varepsilon_{f s} d i s t^{2}\right) \times l, \text { ifdist }<d i s t_{0} \\
e n_{r} \times l+\left(e n_{t}+\varepsilon_{m p} d i s t^{4}\right) \times l, \text { ifdist }>d i s t_{0}
\end{array}\right\}
\end{aligned}
$$

Where $E N_{\text {Trans }}$ represents the complete power transferred from the source to the target to supply a stream of $l$ bits. The $e n_{t}$ and $e n_{r}$ expresses the energy wasted from the radio transmitter and the receiver, during the operation. Then, $\varepsilon_{m p}$ and $\varepsilon_{f s}$ represents the permittivity for a multipath model and for the free space model and it depends on the transmitter amplifier model and dist $t_{0}$ represents the threshold transmission distance.

\subsection{Opposition-based chaotic whale optimization algorithm (OBC-WOA)}

Opposition-based Learning (OBL) has been demonstrated as an efficient technique of improving optimization algorithms in order to overcome certain issues. An estimate $x^{\prime}$ for each alternative $x$ of a specified issue is the primary notion of OBL. We calculate this as follows to discover the contrary value $x^{\prime}$ of the present value $x$ :

$$
x^{\prime}=r+s-x
$$

Where, $x$ is a real number within interval $[\mathrm{r}, \mathrm{s}]$.

Whale Optimization Algorithm (WOA) was implemented by Mirjalili et al. [18] and was a naturally-inspired meta-heuristic optimization algorithm that helped optimize the solution. WOA consists of two stages: exploitation and exploration. Exploration means the worldwide search for optimum alternatives, while local search involves exploitation. The purpose of operation is to examine a limited (and yet promising) search area in order to improve a good 'S' outcome. The WAO algorithm begins with a consistently-generated whale population (solutions). WOA can therefore be regarded as a successful worldwide optimizer. Thus OBC-WOA algorithm develops by introduction of chaos into the WOA algorithm in order to decrease that impact and enhance its effectiveness. Chaotic generally arises from the term ' chaos,' which
Table 1. Chaotic maps

\begin{tabular}{|c|c|}
\hline Name of Map & Equation \\
\hline Bernoulli map & $\begin{array}{c}W_{P+1}=W_{P} / 0.6 \text {,if } 0<W_{p} \leq 0.6 \\
W_{P+1}=\left(W_{p}-0.6\right) / 0.4, \text { if } 0.6<W_{P}<1\end{array}$ \\
\hline Logistic map & $W_{P+1}=4 W_{P}\left(1-W_{P}\right)$ \\
\hline Chebyshev map & $W_{P+1}=\cos \left(0.5 \cos -1\left(W_{P}\right)\right)$ \\
\hline Circle map & $W_{P+1}=W_{P}+0.5-1.1 / \pi \sin (2 \pi$ \\
& $\left.W_{P}\right) \bmod (1)$ \\
\hline Cubic map & $W_{P+1}=2.59 W_{P}\left(1-W_{P}^{2}\right)$ \\
\hline $\begin{array}{c}\text { Iterative chaotic } \\
\text { map with infinite } \\
\text { collapses(ICMIC) } \\
\text { map }\end{array}$ & $W_{P+1}=\sin \left(70 / W_{P}\right)$ \\
\hline Piecewise map & \\
& $=$ \\
\hline Singer map & $W_{P+1}=\left(1-W_{P} / 0.7\right.$, if $W_{P} \in(1-0,0.7)$, if \\
& $W_{P} \in(0.7,1)$ \\
\hline Sinusoidal map & $W_{P+1}=1.073\left(7.86 W_{P}-\right.$ \\
\hline Tent map & $W_{P+1}=2.3 W_{P}{ }^{2} \sin \left(\pi W_{P}\right)$ \\
\hline Sine map & $W_{P+1}=W_{P} / 0.4$, if $0<W_{P} \leq 0.4$ \\
& $W_{P+1}=\left(1-W_{P}\right) / 0.6$, if $0.4<W_{P} \leq 1$ \\
\hline Gaussian map & $W_{P+1}=\sin \left(\pi W_{P}\right)$ \\
\hline & $W_{P+1}=0$, if $W_{P=0}$ \\
& $W_{P+1}=\left(1-W_{P}\right) \bmod (1) W_{P} \neq 0$ \\
\hline
\end{tabular}

implies the possession of a complex system with uncertain behaviour, and maps mean mapping, or assigning chaos to a parameter using a feature. Because of the chaos ' ergodicity and non-repetitive characteristic characteristics, general searches can be performed at greater rates relative to outlets based on probabilities. Chaotic maps in non-linear structures are maps that demonstrate the complicated and vibrant behaviour.

Because of their vibrant behaviour, chaotic maps have been commonly recognized in the sector of optimisation, which helps to enhance optimisation of the search area. The use of chaotic maps can be beneficial to substitute such unpredictability.

A number of chaotic maps with distinct mathematical equations, mentioned in Table 1, are used to bring chaos into the optimization algorithm. The suggested OBC-WOA application is on the static sensor network nodes which are randomly used. $\mathrm{N}$ nodes are supposed to depict the search units of the Cluster top (whales), which are $(\mathrm{CH}=$ $\left.\mathrm{CH}_{1}, \mathrm{CH}_{2} \ldots, \mathrm{CH}_{\mathrm{n}}\right)$. The test respondents' location (applicant $\mathrm{CH}$ ) is displayed in 2D space showing the locations of a node in order to imitate the roles of the search agents (whales) in the OBC-WOA and since the sensor nodes are stationary.

Pseudo code of the proposed OBC-WOA algorithm is illustrated in Algorithm 1. 


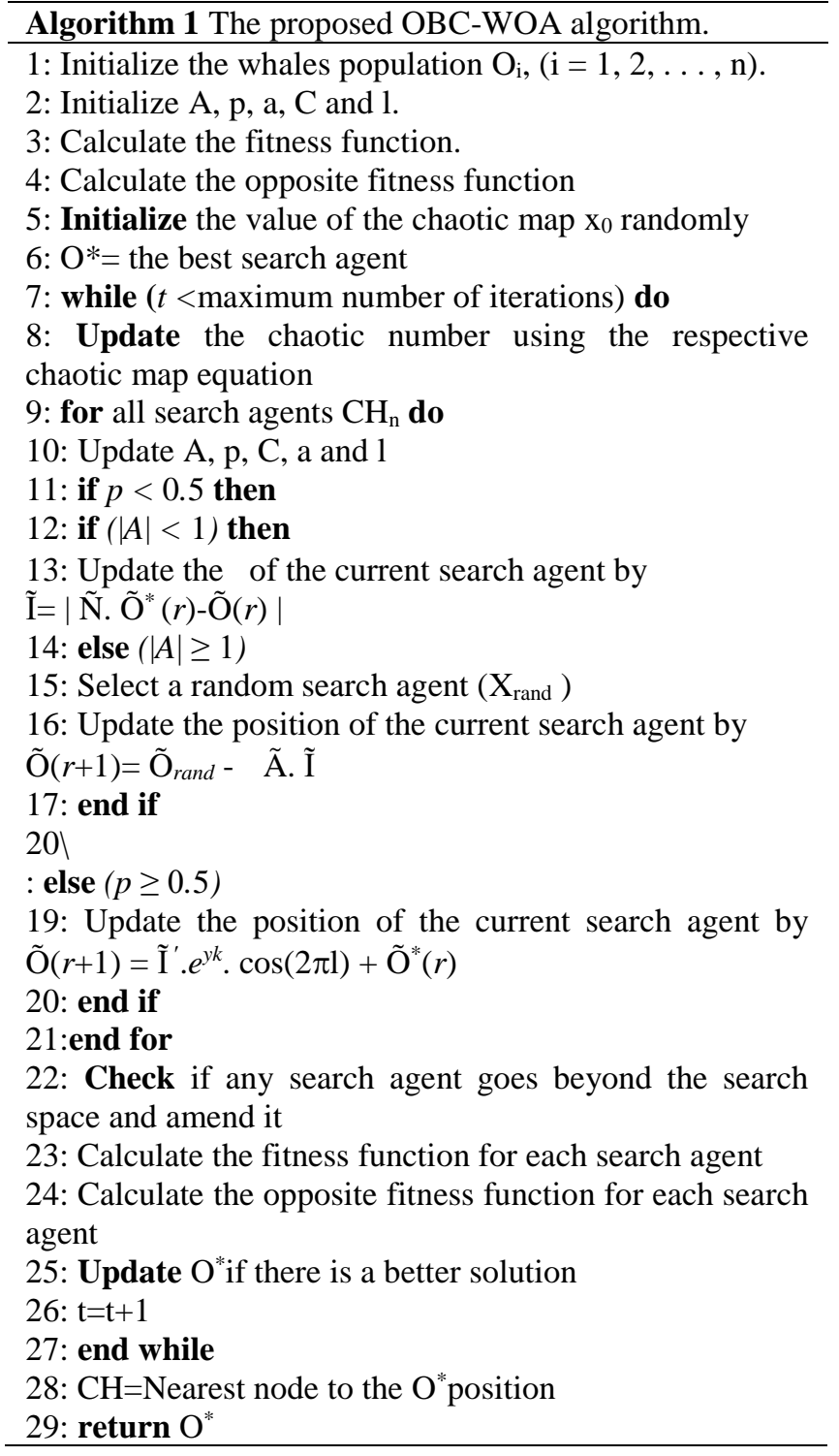

Fig. 2 gives the flowchart for the entire method. A fitness function dictates the decision of the Cluster Head $(\mathrm{CH})$. Fitness plays a vital role when exploring the prey component in the OBC-WOA optimization process. The input is the characteristics of the node, including its residual energy and the amount of neighbours. The fitness function of the $\mathrm{CH}$ node for a cluster $\mathrm{k}$ is given by Eq. (13).

$$
f\left(\mathrm{CH}_{k}\right)=b_{1} \mid N\left(\mathrm{CH}_{k} \mid+b_{2} \sum\left(\mathrm{CH}_{E}\right)\right.
$$

Where $b_{1}$ and $b_{1}$ are selected randomly from 0 to 1. $\mathrm{N}\left(\mathrm{CH}_{k}\right)$ is the list of all plant neighbors in the area of $\mathrm{CH}_{k}$ and $\mathrm{CH}_{E}$ is the remaining power amount of the neighbouring node. The easiest alternative is to have the biggest fitness value and therefore sufficient remaining power and sufficient amount of nearby nodes to become the $\mathrm{CH}$.

Once the base station has identified the optimal



Figure. 2 Flowchart of proposed OBC-WOA approach

configuration of the cluster heads and related group nodes, the basic station transfers data containing the $\mathrm{CH}-\mathrm{ID}$ to each of the network nodes. To arrange information transmission, the chosen cluster head will become a command centre in its vicinity. In addition, the TDMA (Multiple Access Time Division), an itinerary for members of the Cluster, is established to prevent collisions between data members. This also allows the members of the cluster to sleep wake cycle where they only have to be wake in their respective TDMA time slots and conserve energy throughout the cycle of sleep. The node will be in low energy and retain energy during sleeping cycles. At the completion of each round, the cluster top then mixes all the information and transmitted the information merged back to the base station after obtaining information from all the group participants. 
Table 2. WSN Parameters

\begin{tabular}{|c|c|}
\hline Parameter & Value \\
\hline Area of sensor field & $300 \times 300 \mathrm{~m}^{2}$ \\
\hline Base station location & $(100,100) \&(200,200)$ \\
\hline Total number of sensors & 500 \\
\hline Energy of sensor & $1.5 \mathrm{~J}$ \\
\hline Transmitter/ Receiver & $10 \mathrm{~nJ} / \mathrm{bit}$ \\
\hline $\begin{array}{c}\text { Transmit amplifier } / \mathrm{m}^{2} \\
\text { space) }-\varepsilon_{f s}\end{array}$ & $0.0013 \mathrm{pj} / \mathrm{bit} / \mathrm{m}^{4}$ \\
\hline $\begin{array}{c}\text { Transmit amplifier } \\
\text { (multipath)- } \varepsilon_{m p}\end{array}$ & $4000 \mathrm{bits}$ \\
\hline Packet size & $500 \mathrm{bits}$ \\
\hline Message size & $88 \mathrm{~m}$ \\
\hline $\mathrm{d}_{0}$ & $5 \mathrm{~nJ} / \mathrm{bit} / \mathrm{signal}$ \\
\hline $\mathrm{EN}_{\mathrm{DA}}$ & $5 \%$ \\
\hline $\mathrm{b}_{\mathrm{opt}}$ & \\
\hline
\end{tabular}

\section{Simulation results}

\subsection{Environmental setup}

The energy requirements of the set-up stage, information transfer and aggregation are monitored during the simulation. Table 2 lists the parameters of the corresponding simulation.

\subsection{Performance metrics}

\subsubsection{Network lifetime}

Lifetime of the network is described as the amount of cycles to the end of the machine. The network lifetime was measured during the simulation using a single node cycle, i.e. the First Node Death (FND) and the Last Node Death (LND), because the fields within the data collector node coverage can no longer be monitored if a node dies during the data collection process. The amount of deceased nodes vs. cycles can be observed by LND. Lifetime of the network rises as energy consumption rises. (Eq. (6))

\subsubsection{Energy consumption}

The significant energy usage in a specified round can be a reasonable assessment of the power effectiveness of the algorithm. The cumulative quantity of energy left in the battery at the end of the simulation shall be evaluated by calculating the cumulative quantity (in joules) [19, 20]. (Eq. (1))

\subsubsection{Packet delivery ratio}

The delivery rate of the packet can be described as the proportion between the total number of packages achieved and the total number of packets originating at the origin node. (Eq. (9))

\subsubsection{Network throughput}

The network throughput estimates the quantity of helpful information that the BS receives. Network performance is therefore a significant measure for any routing algorithm. (Eq. (8))

\subsection{Performance evaluation}

The performance of OBC-WOA clearly exceeds the performances of the existing method such as FKM-CMA [17], WOA [18], PSO [12], and GSA [20]. Fig. 3 shows a complete network power usage at 300 nodes and 24 round gateways. By decreasing the distance between sensors to gateway and gateway to sensors, OBC-WOA saves more energy compared to other existing methods such as WOA, PSO, GSA and FKM-CMA. The WOA and GSA shape the clusters efficiently, however, in assigning the member devices to the gateways and thereby decreasing the energy consumption.
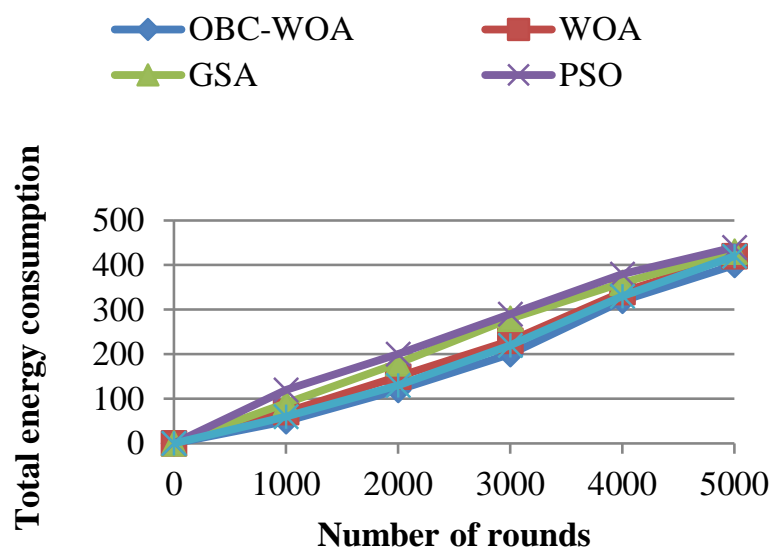

Figure. 3 Total energy consumption of WSN
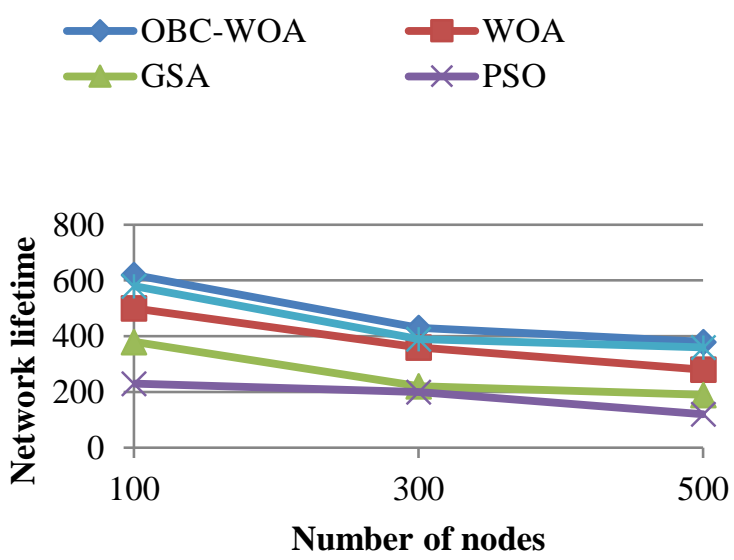

Figure. 4 Network lifetime of WSN 


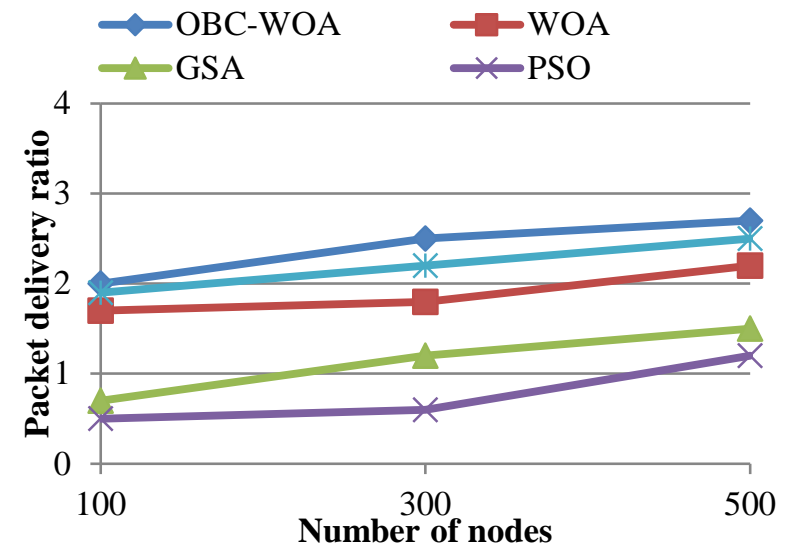

Figure. 5 Packet delivery ratio of WSN

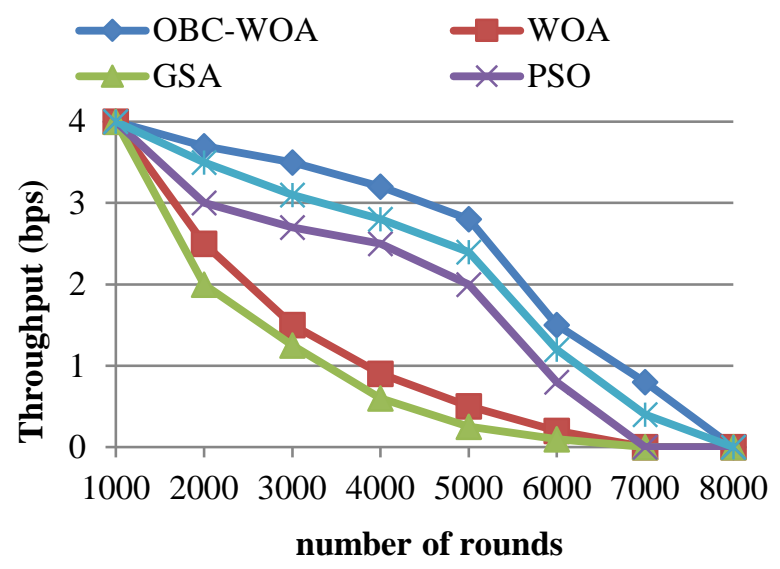

Figure. 6 Throughputs in WSN

Fig. 4 together with multiple sensor nodes, illustrates the network life. Thus, the network lifetime in OBC-WOA is higher than the existing algorithms WOA, GSA, FKM-CMA and PSO. The reason for this is to use the novel fitness function to take care of the gateways sensors.

Fig. 5 shows the efficiency with respect to the packets obtained by the BS of the suggested algorithm. The fact that compared with WOA, BSD, GSA and PSO algorithms, OBC-WOA has obtained more detected packages. Simulations will be conducted to compare the performance through various algorithms like OBC-WOA, WOA, GSA and PSO.

Table 3. Overall performance of WSN

\begin{tabular}{|c|c|c|c|}
\hline $\begin{array}{c}\text { Number of } \\
\text { sensors }= \\
\mathbf{5 0 0}\end{array}$ & FND & LND & $\begin{array}{c}\text { Throughput at } \\
\text { round 4000 (bits } \\
\text { /round) }\end{array}$ \\
\hline OBC-WOA & 3045 & 8300 & 435000 \\
\hline WOA & 2482 & 7268 & 415200 \\
\hline GSA & 980 & 4962 & 32000 \\
\hline PSO & 1949 & 6639 & 396000 \\
\hline
\end{tabular}

The output is defined as the total number of packets the BS receives at a given moment. The performance is calculated for WSN with 500 nodes and $25 \mathrm{CHs}$ and shown in Fig. 6.

As it utilizes less energy, the suggested algorithm has a longer network life. The network performance is equal to the overall number of live nodes in the network, which usually gives BS a better performance in the central positioning of the network. Thus, the throughput of OBC-WOA exceeds compared to other existing methods like WOA, PSO, GSA and BSD. Table 3 gives the general efficiency of the algorithm and a quality of around 4000.

\section{Conclusion and future scope}

In summary, this analysis introduces a comprehensive energy-efficient chaotic Whale Optimization Routing Algorithm (WOA) that allows you to pick cluster heads for optimal use of power in WSN. The Tent map has significantly increased WOA's efficiency among all the chaotic maps. The simulation results show that the proposed method is $12 \%$ better than Whale Optimization algorithm (WOA), 25\% better than Gravitational Search Algorithm (GSA), 40\% better than Particle Swarm Optimisation (PSO) and 8\% better than Fuzzy KMeans and Centralized Mid-point Algorithm (FKMCMA). As compared with the existing methods, the convergence speed should be improved with less energy consumption and thereby improving the overall performances. The scientific contribution of the proposed method is to increase the solution precision and reliability by using Opposition-based Chaotic Whale Optimization (OBC-WOA). OBCWOA works better than the algorithms WOA, GSA and PSO. The reason for this is to use the novel fitness function to take care of the gateways sensors. Future work aims at developing an effective routing algorithm, taking into account other variables such as energy balance in the fitness function.

\section{References}

[1] A. R. Jadhav and T. Shankar, "Whale optimization based energy-efficient cluster head selection algorithm for wireless sensor networks", International Journal of Neural and Evolutionary Computing, pp.1-22, 2017, arXiv preprint arXiv: 1711.09389

[2] P. Goel and Er. A. Goyal, "Clustering in Wireless Sensor Networks: Survey", International Journal of Advance Research and Innovative Ideas in Education, Vol. 3, No.5, pp. 361-365, 2017. 
[3] K. Muthukumaran, K. Chitra, and C. Selvakumar, "Energy Efficient Clustering in wireless sensor networks", In: Proc. of International Conf. on Inventive Computing and Informatics (ICICI), pp.1-5, 2017.

[4] Z. Zhao, K. Xu, G. Hui, and L. Hu, "An Energy-Efficient Clustering Routing Protocol for Wireless Sensor Networks Based on AGNES with Balanced Energy Consumption Optimization", International Journal of Sensors, Vol. 18, No. 11, pp. 1-27, 2018.

[5] P. C. S. Rao, H. Banka, and P. K. Jana, "Energy Efficient Clustering for Wireless Sensor Networks: A Gravitational Search Algorithm", International Journal of Lecture Notes in Computer Science, Vol. 9873, No.1, pp. 247259, 2016.

[6] G. Kaur and S. Arora, "Chaotic whale optimization algorithm", International Journal of Computational Design and Engineering, Vol. 5, No.3, pp. 275-284, 2018.

[7] P. Mann and S. Singh, "Artificial bee colony metaheuristic for energy-efficient clustering and routing in wireless sensor networks", International Journal of Soft Computing, Vol. 21, No. 22, pp. 6699-6712, 2016.

[8] M. Ahmed, E. Houssein, A. Hassanien, A. Taha, and E. Hassanien, "Maximizing lifetime of large-scale wireless sensor networks using multi-objective whale optimization algorithm", International Journal of Telecommunication Systems, pp. 1-17, 2019.

[9] I. Zaatouri, A. B. Guiloufi, N. Alyaoui, and A. Kachouri, "A Comparative Study of the Energy Efficient Clustering Protocols in Heterogeneous and Homogeneous Wireless Sensor Networks", Wireless Personnel Communications, Vol. 97, No,.4, pp. 6453-6468, 2017.

[10] V. Saranya, S. Shankar, and G. R. Kanagachidambaresan, "Energy Efficient Clustering Scheme (EECS) for Wireless Sensor Network with Mobile Sink", International Journal of Wireless Personnel Communications, Vol. 100, No.4, pp. 1553-1567, 2018.

[11] Q. Wang, S. Guo, J. Hu, and Y. Yang, "Spectral partitioning and fuzzy C-means based clustering algorithm for big data wireless sensor networks", EURASIP Journal on Wireless Communications and Networking, Vol. 54, No.1, pp. 1-11, 2018.

[12] M. Azharuddin and P. K. Jana, "PSO-based approach for energy-efficient and energybalanced routing and clustering in wireless sensor networks", International Journal of Soft
Computing, Vol. 21, No.22, pp. 6825-6839, 2017.

[13] P. Lalwani, S. Das, H. Banka, and C. Kumar, "CRHS: Clustering and routing in wireless sensor networks using harmony search algorithm", International Journal of Neural Computing and Applications, Vol. 30, No. 2, pp. 639-659, 2018.

[14] O. M. D. Alia, "A dynamic harmony searchbased fuzzy clustering protocol for energyefficient wireless sensor networks", International Journal of Annals of Telecommunications, Vol. 73, No.5-6, pp. 353365, 2018.

[15] A. Zahedi, M. Arghavani, F. Parandin, and A. Arghavani, "Energy Efficient ReservationBased Cluster Head Selection in WSNs", International Journal of Wireless Personnel Communications, Vol.100, No.3, pp. 667-679, 2018.

[16] S. Dutt, S. Agrawal, and R. Vig, "ClusterHead Restricted Energy Efficient Protocol (CREEP) for Routing in Heterogeneous Wireless Sensor Networks", International Journal of Wireless Personnel Communications, Vol.100, No.4, pp. 1477-1497, 2018.

[17] N. Srikanth and M.S.G. Prasad, "Efficient Clustering Protocol Using Fuzzy K-means and Midpoint Algorithm for Lifetime Improvement in WSNs", International Journal of Intelligent Engineering and Systems, Vol.11, No.4, pp.6171, 2018.

[18] S. Mirjalili and A. Lewis, "The whale optimization algorithm", International Journal of Advances in Engineering Software, Vol. 95, No. 5, pp. 1-67, 2016.

[19] A. Rostami and M. H. Mottar, "Wireless Sensor Network Clustering Using Particles Swarm Optimization for Reducing Energy Consumption", International Journal of Managing Information Technology (IJMIT), Vol. 6, No. 4, pp. 1-15, 2014.

[20] T. Wang, G. Zhang, X. Yang, and A. Vajdi, "Genetic Algorithm for Energy-Efficient Clustering and Routing in Wireless Sensor Networks", International Journal of Systems \& Software, Vol. 146, No.12, pp. 196-214, 2018. 